\title{
Morphological and Chemical Mapping of Columnar Lithium Metal SUPPLEMENTARY INFORMATION
}

Wesley Chang ${ }^{1,3,5,6}$, Dr. Jeung Hun Park ${ }^{1,2,3,5}$, Nikita S. Dutta ${ }^{1}$, Prof. Craig B. Arnold ${ }^{1,2,3}$, Dr. Daniel A. Steingart $1,2,3,4,5,6, *$

${ }^{1}$ Department of Mechanical and Aerospace Engineering, ${ }^{2}$ Department of Chemical and Biological Engineering, and ${ }^{3}$ Andlinger Center for Energy and the Environment, Princeton University, Princeton, NJ 08540 USA

${ }^{4}$ Department of Earth and Environmental Engineering, ${ }^{5}$ Department of Chemical Engineering, and ${ }^{6}$ Columbia Electrochemical Energy Center, Columbia University, New York, NY 10027 USA

*Correspondence: dan.steingart@columbia.edu 
All capacities and current densities are areal $\left(\right.$ per $\left.\mathrm{cm}^{2}\right)$.
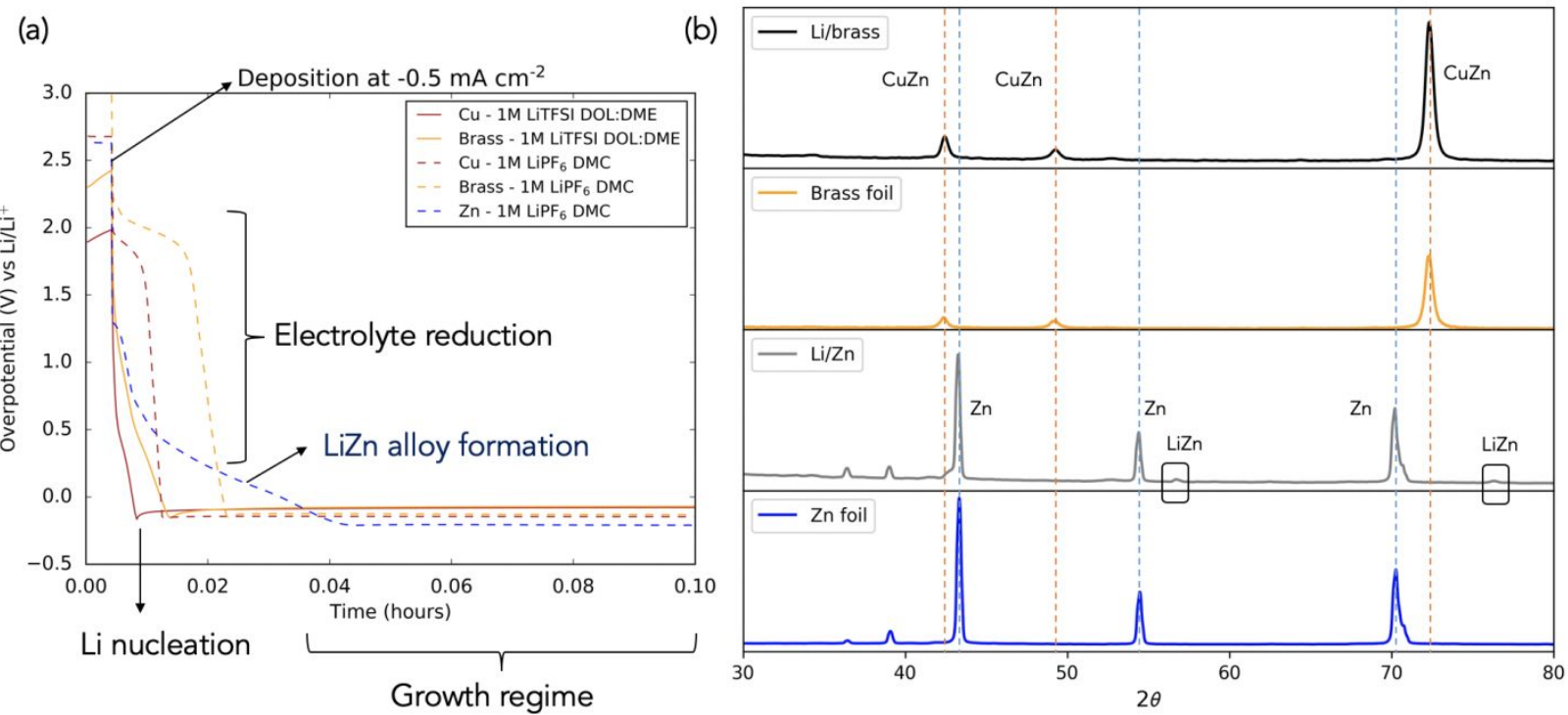

Figure S1. (a) Deposition overpotential (vs Li/Li ${ }^{+}$) profiles for Li plating on either $\mathrm{Cu}$ and brass, in either $1 \mathrm{M}$ LiTFSI DOL:DME and $1 \mathrm{M} \mathrm{LiPF}_{6}$ DMC, and Li plating on $\mathrm{Zn}$ in $1 \mathrm{M} \mathrm{LiPF} 6 \mathrm{DMC}$ All four profiles for $\mathrm{Cu}$ and brass substrates show initial electrolyte reduction and a nucleation peak followed by long-term growth. Deposition on $\mathrm{Zn}$ foil shows alloying behavior with no nucleation peak. All depositions were carried out at $-0.5 \mathrm{~mA} \mathrm{~cm}^{-2}$. (b) Corresponding XRD spectra for $\mathrm{Li}$ deposited on brass and $\mathrm{Zn}$ foil, and brass and zinc foil reference. LiZn peaks can be observed for Li deposited on $\mathrm{Zn}$ (see black square), but not for Li deposited on brass.

(a)

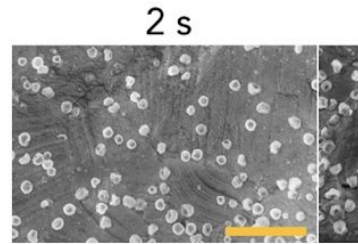
$10 \mathrm{~s}$ $1 \mathrm{~min}$ $5 \mathrm{~min}$ $15 \min$
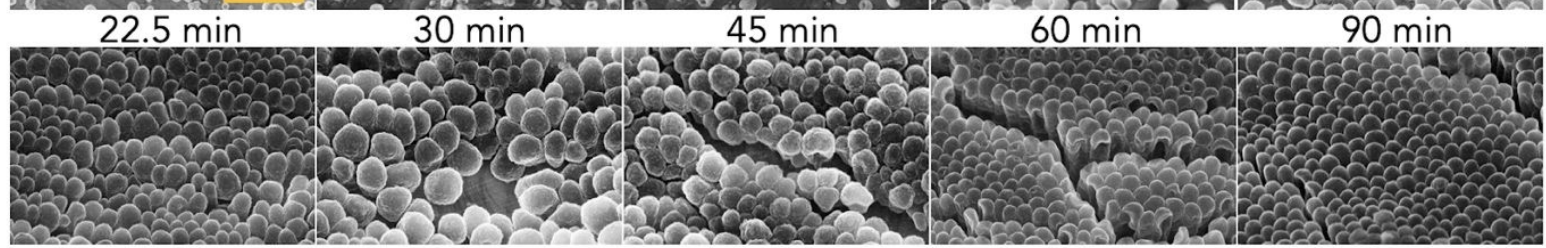

(b)
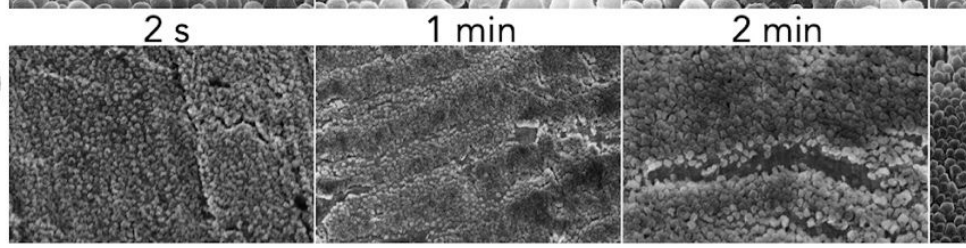

$15 \mathrm{~min}$

$60 \mathrm{~min}$

Figure S2. Growth evolution of columnar Li on (a) acid treated stainless steel 321 and (b) acid treated brass $\left(\mathrm{Cu}_{63} \mathrm{Zn}_{37}\right)$, at labeled time intervals. Each image was taken after a separate plating carried out at the labeled time interval at $0.5 \mathrm{~mA} \mathrm{~cm}^{-2}$. All images are at the same magnification, 
with the scale bar representing $1 \mu \mathrm{m}$. The columnar diameters for brass are noticeably smaller than the diameters for stainless steel. Statistical data on diameters for various substrates are provided in Figure 1. It should also be observed that columnar morphology (growth in vertical direction) starts to appear before the plating becomes completely close-packed. This indicates that the columnar growth may be determined by preferred deposition sites on pre-existing nuclei (an electrochemical effect), rather than a mechanical effect due to SEI formation causing nuclei to impinge upon each other (as suggested by Zhang et al.).

(a) +0.05 mAh (at $4 \mathrm{~mA}$ )

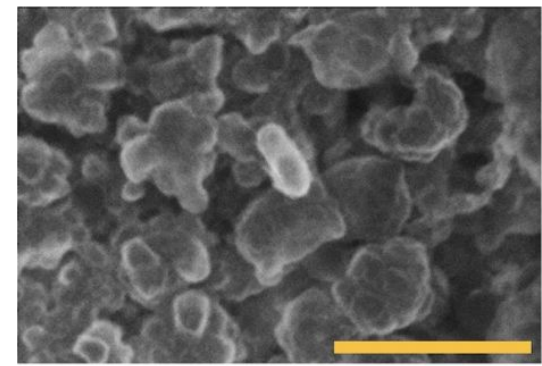

(b) +0.13 mAh (at $4 \mathrm{~mA})$

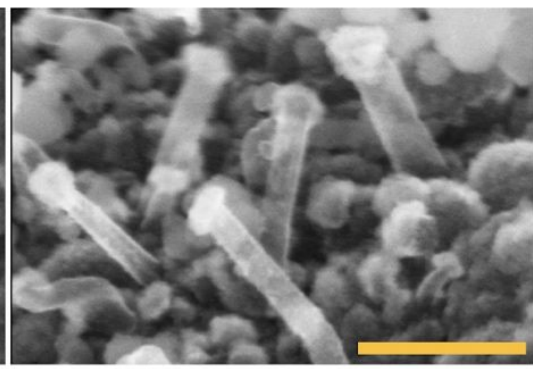

(c) $-0.05 \mathrm{mAh}($ at $4 \mathrm{~mA})$

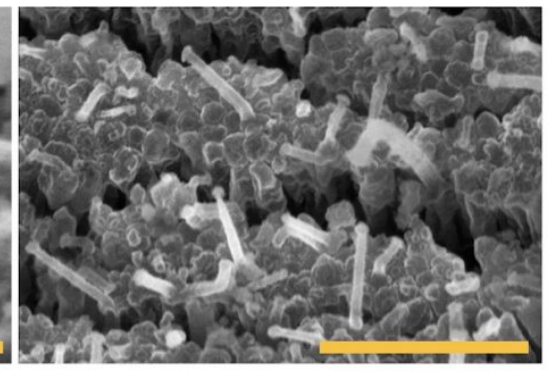

Figure S3. High resolution SEM images of growth protrusions on columnar lithium deposits in $1 \mathrm{M} \mathrm{LiPF}_{6} \mathrm{DMC}_{\text {, at (a) } 4 \mathrm{~mA} \mathrm{~cm}}^{-2}, 0.050 \mathrm{mAh} \mathrm{cm}^{-2}$; (b) $4 \mathrm{~mA} \mathrm{~cm}^{-2}, 0.133 \mathrm{mAh} \mathrm{cm}^{-2}$; and (c) after $0.050 \mathrm{mAh} \mathrm{cm}^{-2} \mathrm{Li}$ stripped. Scale bars indicate (a) $200 \mu \mathrm{m}$, (b) $300 \mu \mathrm{m}$, (c) $5 \mu \mathrm{m}$.

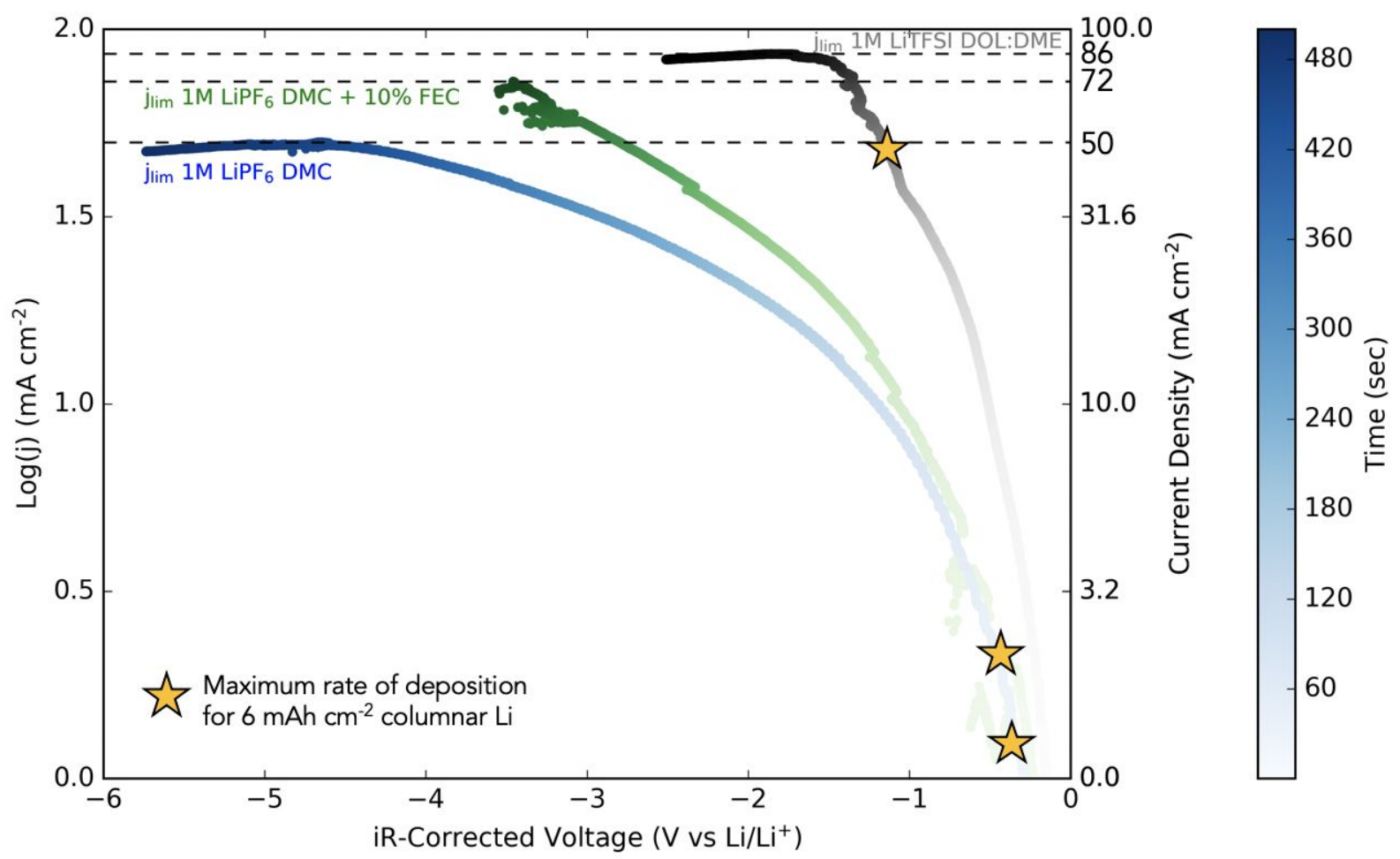

Figure S4. Linear sweep voltammetry from $0 \mathrm{~V}$ to $-10 \mathrm{~V}$ vs reference Li for deposition on brass substrate in the three electrolytes, used to plot the depicted logarithmic current density on the $y-$ axis $\left(\mathrm{mA} \mathrm{cm}^{-2}\right)$ and $\mathrm{iR}$-corrected voltage $\left(\mathrm{V}\right.$ vs $\left.\mathrm{Li} / \mathrm{Li}^{+}\right)$on the $\mathrm{x}$-axis. iR-correction was done by using the ohmic resistance calculated by EIS, as shown in Figure S5. As shown, the current 
densities for columnar growth in all three electrolytes is below their respective maximum current densities.

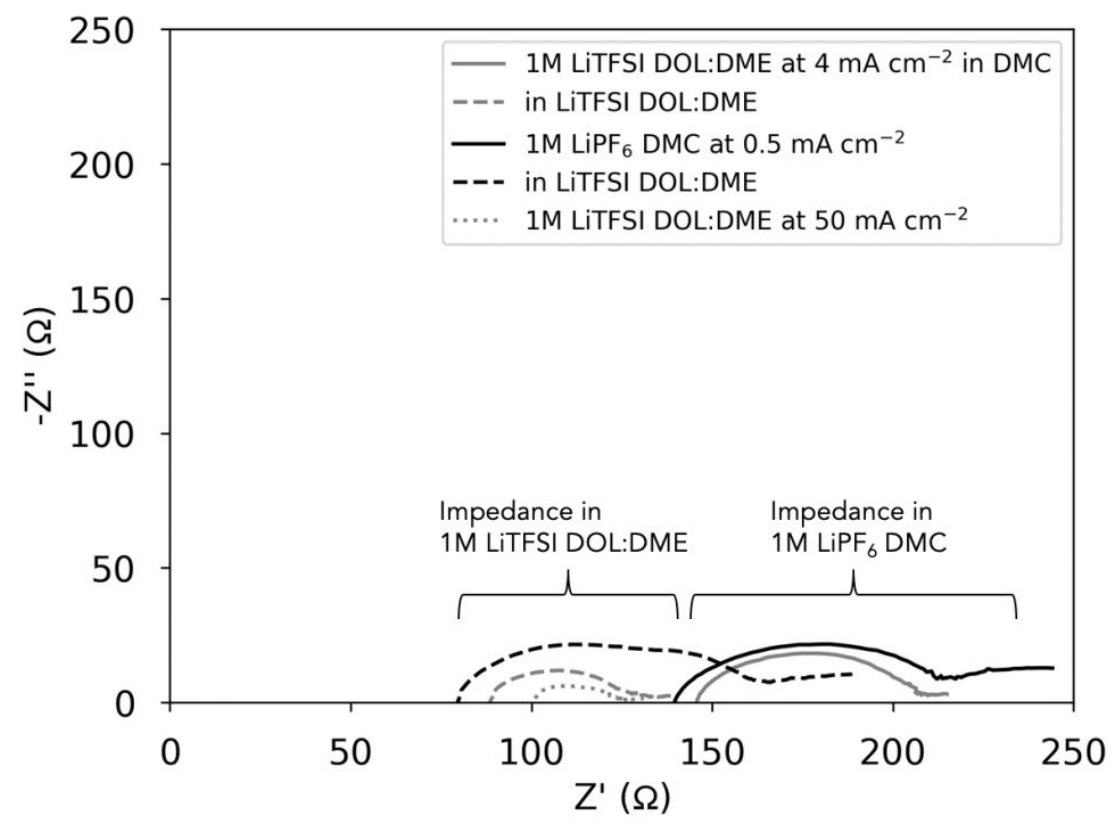

Figure S5. Columnar Li deposited in either 1M LiTFSI DOL:DME (gray) or 1M LiPF 6 DMC (black), with subsequent electrochemical impedance spectroscopy in either 1M LiTFSI

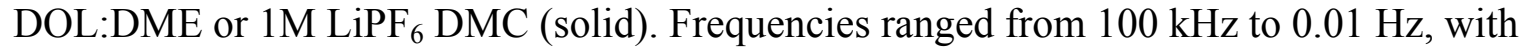
voltage amplitude of $0.1 \mathrm{~mA}$ and 14 points per decade. The x-intercept of the high frequency indicates the ohmic resistance, this indicates that 1M LiTFSI DOL:DME has lower ohmic resistance than $1 \mathrm{M} \mathrm{LiPF} 6$ DMC.

(a) $0.5 \mathrm{~mA}$ (at $0.5 \mathrm{mAh}$ )

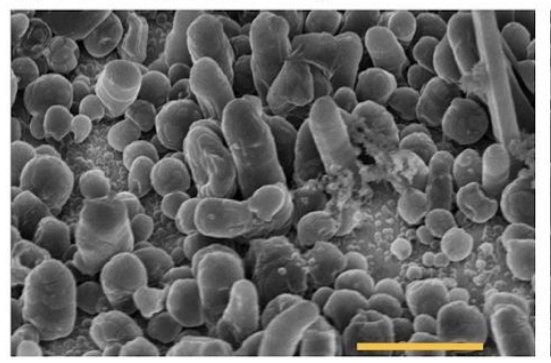

(b) $1 \mathrm{~mA}$ (at $0.5 \mathrm{mAh}$ )

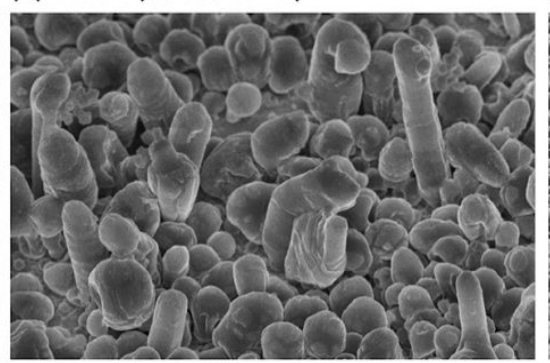

(c) $4 \mathrm{~mA}$ (at $0.5 \mathrm{mAh}$ )

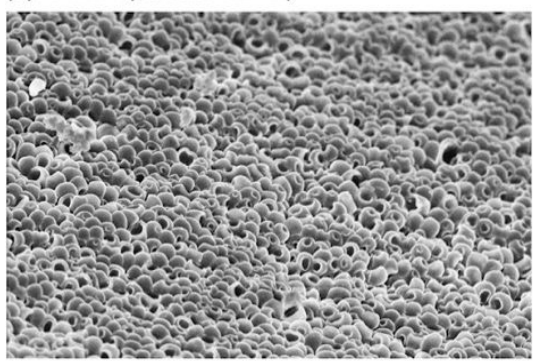

Figure S6. Deposition in 1M LiTFSI DOL:DME at (a) $0.5 \mathrm{~mA} \mathrm{~cm}^{-2}$, (b) $1 \mathrm{~mA} \mathrm{~cm}^{-2}$, and (c) 4 $\mathrm{mA} \mathrm{cm}{ }^{-2}$. All plating tests were done with a total plating capacity of $0.5 \mathrm{mAh} \mathrm{cm}^{-2}$. Scale bar corresponds to $5 \mu \mathrm{m}$. 


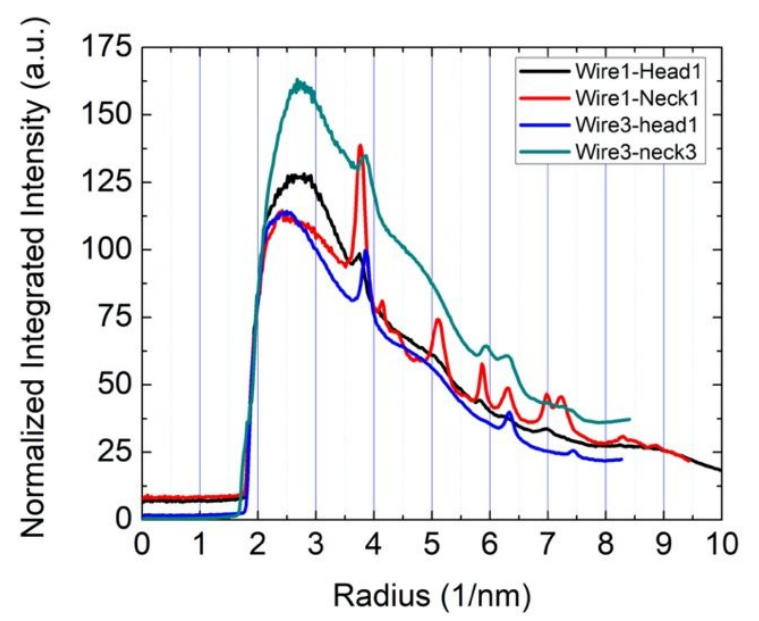

\begin{tabular}{|r|r|l|l|l|l|l|l|}
\hline $\begin{array}{l}\text { Wire1 } \\
\text { head1 }\end{array}$ & $\begin{array}{l}\text { D-spacing } \\
\text { (Angstrom }\end{array}$ & Li & $\mathrm{LiF}$ & $\mathrm{Li}_{2} \mathrm{O}$ & $\mathrm{Li}_{2} \mathrm{O}_{2}$ & $\mathrm{Li}_{2} \mathrm{CO}_{3}$ & LiOH* $\mathrm{H}_{2} \mathrm{O}$ \\
\hline 1 & 2.65 & & & $\mathrm{Li}_{2} \mathrm{O}(111)$ & & $\begin{array}{l}\mathrm{Li}_{2} \mathrm{CO}_{3}(- \\
112)\end{array}$ & $\mathrm{LiOH} * \mathrm{H}_{2} \mathrm{O}(220)$ \\
\hline 2 & 2.38 & & $\mathrm{LiF}(111)$ & & & & \\
\hline 3 & 1.7 & & & & & & \\
\hline 4 & 1.58 & & & & $\mathrm{Li}_{2} \mathrm{O}_{2}(110)$ & & \\
\hline 5 & 1.43 & $\mathrm{Li}(211)$ & $\mathrm{LiF}(220)$ & & & & \\
\hline 6 & 1.2 & & & & $\mathrm{Li}_{2} \mathrm{O}_{2}(203)$ & & \\
\hline 7 & 1.15 & & $\mathrm{LiF}(222)$ & & & & \\
\hline 8 & 1.1 & $\mathrm{Li}(310)$ & & & $\mathrm{Li}_{2} \mathrm{O}_{2}(204)$ & & \\
\hline
\end{tabular}

\begin{tabular}{|c|c|c|c|c|c|c|c|}
\hline $\begin{array}{l}\text { Wire1 } \\
\text { - } \\
\text { neck1 }\end{array}$ & $\begin{array}{l}\text { D-spacing } \\
\text { (Angstrom } \\
\text { ) }\end{array}$ & $\mathrm{Li}$ & $\mathrm{LiF}$ & $\mathrm{Li}_{2} \mathrm{O}$ & $\mathrm{Li}_{2} \mathrm{O}_{2}$ & $\mathrm{Li}_{2} \mathrm{CO}_{3}$ & $\mathrm{LiOH}^{*} \mathrm{H}_{2} \mathrm{O}$ \\
\hline 1 & 4.22 & & & & & $\begin{array}{l}\mathrm{Li}_{2} \mathrm{CO}_{3}(110 \\
\end{array}$ & \\
\hline 2 & 2.65 & & & $\mathrm{Li}_{2} \mathrm{O}(111)$ & & $\begin{array}{l}\mathrm{Li}_{2} \mathrm{CO}_{3}(- \\
112)\end{array}$ & $\mathrm{LiOH}^{*} \mathrm{H}_{2} \mathrm{O}(220)$ \\
\hline 3 & 2.4 & & $\begin{array}{l}\mathrm{LiF} \\
(111) \\
\end{array}$ & & & & \\
\hline 4 & 1.95 & & & & & & $\mathrm{LiOH}^{*} \mathrm{H}_{2} \mathrm{O}(-301)$ \\
\hline 5 & 1.78 & $\operatorname{Li}(200)$ & & & & & \\
\hline 6 & 1.7 & & & & & & \\
\hline 7 & 1.58 & & & & $\mathrm{Li}_{2} \mathrm{O}_{2}(110)$ & & \\
\hline 8 & 1.43 & $\operatorname{Li}(211)$ & $\operatorname{LiF}(220)$ & & & & \\
\hline 9 & 1.37 & & & $\mathrm{Li}_{2} \mathrm{O}(311)$ & & & \\
\hline
\end{tabular}




\begin{tabular}{|c|c|c|c|c|c|c|c|}
\hline $\begin{array}{l}\text { Wire3 } \\
- \\
\text { head2 }\end{array}$ & $\begin{array}{l}\text { D-spacing } \\
\text { (Angstrom } \\
\text { ) }\end{array}$ & $\mathrm{Li}$ & $\mathrm{LiF}$ & $\mathrm{Li}_{2} \mathrm{O}$ & $\mathrm{Li}_{2} \mathrm{O}_{2}$ & $\mathrm{Li}_{2} \mathrm{CO}_{3}$ & $\begin{array}{l}\mathrm{LiOH} \text { or } \\
\mathrm{LiOH}^{*} \mathrm{H}_{2} \mathrm{O}\end{array}$ \\
\hline 1 & 2.63 & & & $\mathrm{Li}_{2} \mathrm{O}(111)$ & & $\mathrm{Li}_{2} \mathrm{CO}_{3}(-112)$ & $\begin{array}{l}\mathrm{LiOH}^{*} \mathrm{H}_{2} \mathrm{O}(22 \\
0)\end{array}$ \\
\hline 2 & 2.56 & & & & $\mathrm{Li}_{2} \mathrm{O}_{2}(101)$ & & \\
\hline 3 & 2.38 & & $\begin{array}{l}\mathrm{LiF} \\
(111)\end{array}$ & & & & \\
\hline 4 & 1.67 & & & & & & \\
\hline 5 & 1.58 & & & & $\mathrm{Li}_{2} \mathrm{O}_{2}(110)$ & & \\
\hline 6 & 1.34 & & & & $\mathrm{Li}_{2} \mathrm{O}_{2}(105)$ & & \\
\hline
\end{tabular}

\begin{tabular}{|c|c|c|c|c|c|c|c|}
\hline $\begin{array}{l}\text { Wire3 } \\
- \\
\text { neck3 }\end{array}$ & $\begin{array}{l}\text { D-spacing } \\
\text { (Angstrom } \\
\text { ) }\end{array}$ & $\mathrm{Li}$ & $\mathrm{LiF}$ & $\mathrm{Li}_{2} \mathrm{O}$ & $\mathrm{Li}_{2} \mathrm{O}_{2}$ & $\mathrm{Li}_{2} \mathrm{CO}_{3}$ & $\begin{array}{l}\mathrm{LiOH} \text { or } \\
\mathrm{LiOH}^{*} \mathrm{H}_{2} \mathrm{O}\end{array}$ \\
\hline 1 & 2.86 & & & & & $\mathrm{Li}_{2} \mathrm{CO}_{3}(002)$ & \\
\hline 2 & 2.78 & & & & & & $\mathrm{LiOH}(101)$ \\
\hline 3 & 2.58 & & & & $\mathrm{Li}_{2} \mathrm{O}_{2}(101)$ & & \\
\hline 4 & 1.69 & & & & & & \\
\hline 5 & 1.6 & & & & & $\mathrm{Li}_{2} \mathrm{CO}_{3}(-130)$ & \\
\hline 6 & 1.56 & & & & $\mathrm{Li}_{2} \mathrm{O}_{2}(110)$ & & \\
\hline 7 & 1.42 & $\begin{array}{l}\operatorname{Li}(211 \\
)\end{array}$ & $\begin{array}{l}\operatorname{LiF}(220 \\
)\end{array}$ & & & & \\
\hline 8 & 1.33 & & & & $\mathrm{Li}_{2} \mathrm{O}_{2}(105)$ & & \\
\hline
\end{tabular}

Figure S7. Diffraction peak indexing using normalized integrated intensity. Peak positions from reference JC-PDS Catalogue \#45-1460 (for LiF), \#15-0401 (for Li), \#09-0359 (for $\mathrm{Li}_{2} \mathrm{CO}_{3}$ ), \#250486 (for $\left.\mathrm{LiOH}^{*} \mathrm{H}_{2} \mathrm{O}\right), \# 12-0254\left(\mathrm{Li}_{2} \mathrm{O}\right)$. For Figure 5, growth asperities at $2 \mathrm{~mA} \mathrm{~cm}{ }^{-2}$ in $1 \mathrm{M}$ $\mathrm{LiPF}_{6} \mathrm{DMC}$. 

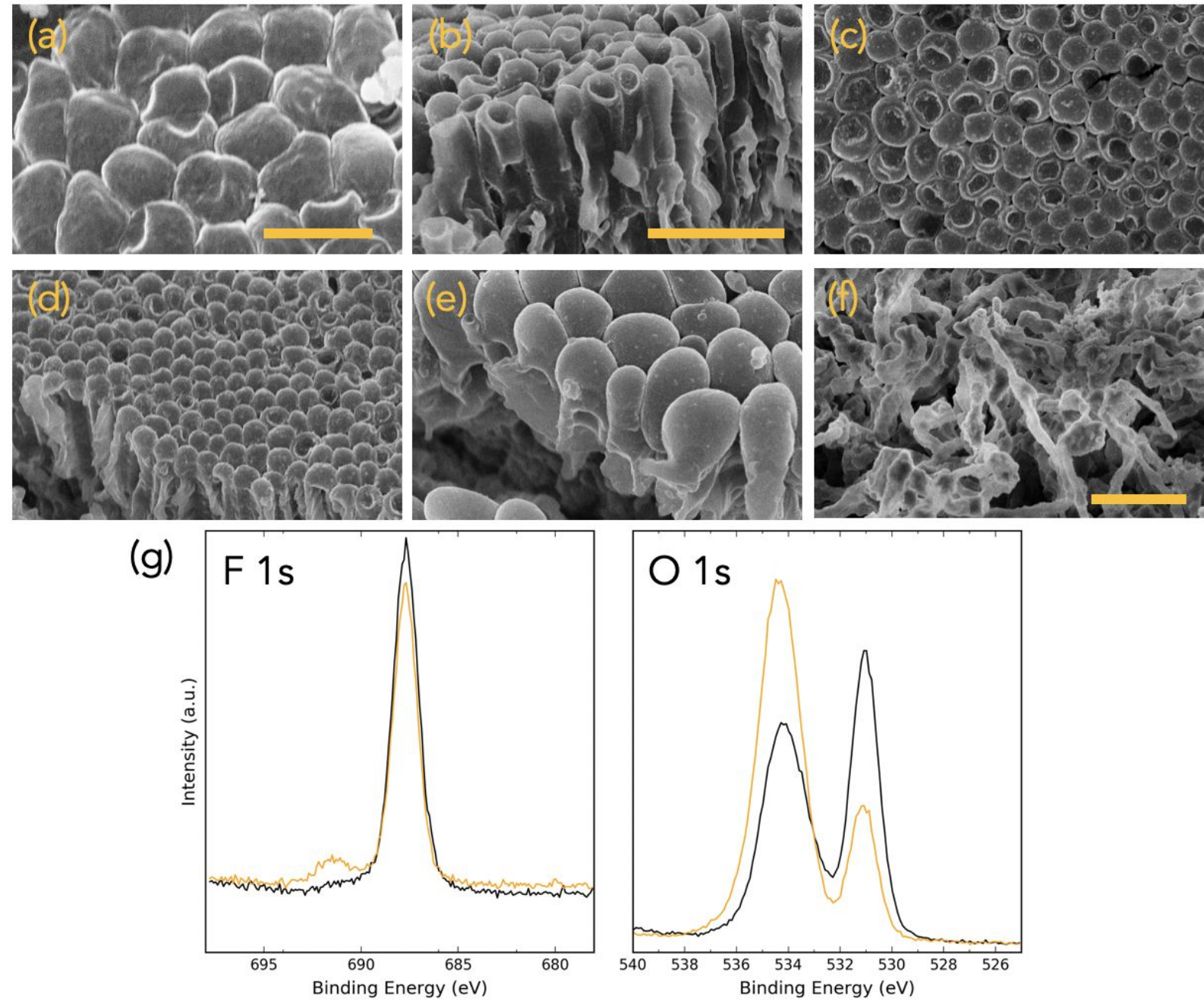

Figure S8. Plating in 1M LiTFSI DOL:DME at (a) $16 \mathrm{~mA} \mathrm{~cm}^{-2}$, (b) $20 \mathrm{~mA} \mathrm{~cm}^{-2}$, (c) $24 \mathrm{~mA} \mathrm{~cm}^{-2}$, (d) $40 \mathrm{~mA} \mathrm{~cm}^{-2}$, (e) $50 \mathrm{~mA} \mathrm{~cm}^{-2}$, and (f) $60 \mathrm{~mA} \mathrm{~cm}^{-2}$, for a total of $2 \mathrm{mAh} \mathrm{cm}^{-2}(10 \mu \mathrm{m}$ thickness). (g) Corresponding XPS point scans for $\mathrm{F} 1 \mathrm{~s}$ and $\mathrm{O} 1 \mathrm{~s}$ scans, with black curves representing uniform plating at $4 \mathrm{~mA} \mathrm{~cm}^{-2}$ and orange curves representing non-uniform plating at $60 \mathrm{~mA} \mathrm{~cm}^{-2}$. 

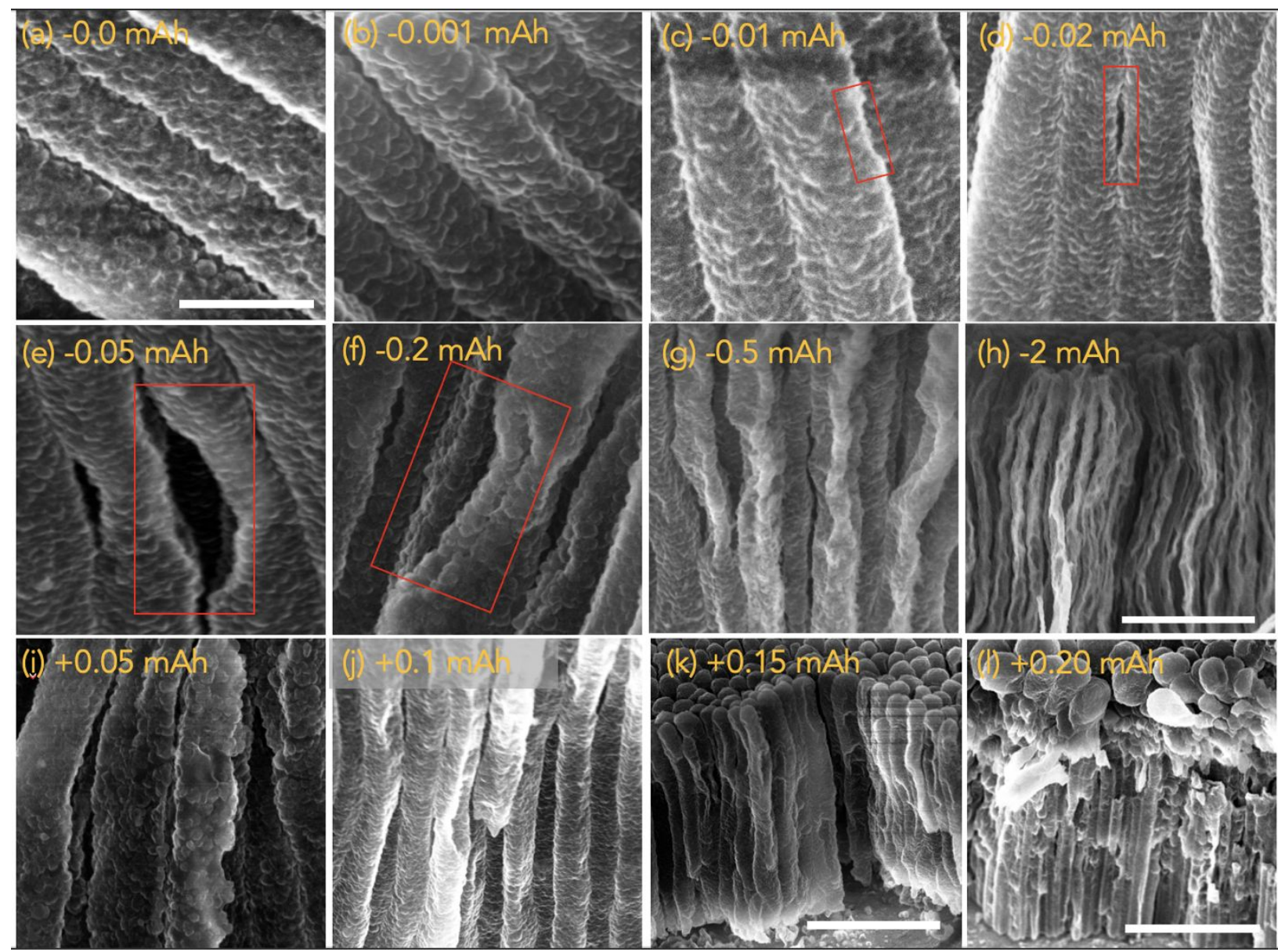

Figure S9. High resolution images of cross-sectional view during stripping and replating, after initial plating of $0.5 \mathrm{~mA} \mathrm{~cm} \mathrm{c}^{-2}, 1 \mathrm{mAh} \mathrm{cm} \mathrm{m}^{-2}$ columnar lithium. After initial plating of columnar lithium, stripping indicates that the thickness/height of the deposits does not change. Rather, lithium is stripped from various locations along the column, indicating a high electrochemically active surface area along the entire lengths of the columns. (h), (k), (l) scale bars correspond to 3 $\mu \mathrm{m}$, all other subplot scale bars correspond to $500 \mathrm{~nm}$. cycled at $2 \mathrm{~mA} \mathrm{~cm}^{-2}$

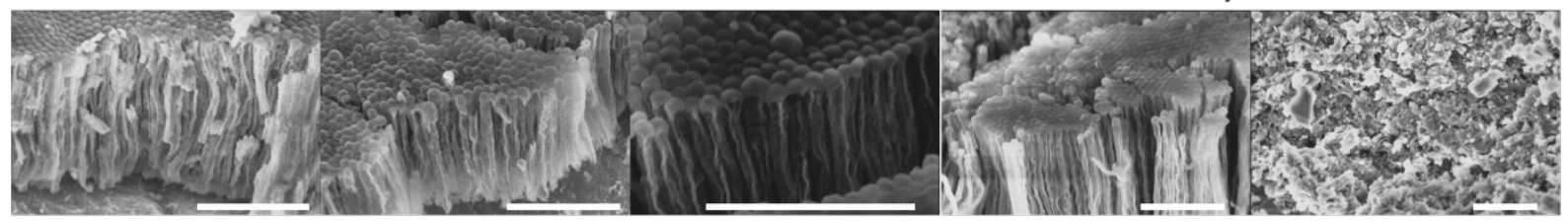

(b) $1 \mathrm{mAh} \mathrm{cm}^{-2}$ in DMC $+10 \% \mathrm{FEC}$

(d) $6 \mathrm{mAh} \mathrm{cm}^{-2}$ in DMC $+10 \% \mathrm{FEC}$

Figure S10. Post-mortem SEM images of (a) $1 \mathrm{mAh} \mathrm{cm}^{-2}$ plated columnar Li in $1 \mathrm{M} \mathrm{LiPF} 6 \mathrm{DMC}_{\text {, }}$ (b) $1 \mathrm{mAh} \mathrm{cm}^{-2}$ plated columnar Li in $1 \mathrm{M} \mathrm{LiPF}_{6} \mathrm{DMC}+10 \% \mathrm{FEC}$, (c) $2 \mathrm{mAh} \mathrm{cm}{ }^{-2}$ plated columnar $\mathrm{Li}$ in $1 \mathrm{M} \mathrm{LiPF}_{6} \mathrm{DMC}+10 \% \mathrm{FEC}$, (d) $6 \mathrm{mAh} \mathrm{cm}^{-2}$ plated columnar Li in $1 \mathrm{M} \mathrm{LiPF}_{6}$ $\mathrm{DMC}+10 \% \mathrm{FEC}$, and (e) $6 \mathrm{mAh} \mathrm{cm}^{-2}$ plated columnar $\mathrm{Li}$ in $1 \mathrm{M} \mathrm{LiPF} 6 \mathrm{DMC}^{-10 \% \mathrm{FEC} \text { and }}$ cycled at higher current of $2 \mathrm{~mA} \mathrm{~cm}{ }^{-2}, 1 \mathrm{mAh} \mathrm{cm}^{-2}$ (all other conditions were cycled at $0.5 \mathrm{~mA}$ $\mathrm{cm}^{-2}, 1 \mathrm{mAh} \mathrm{cm}^{-2}$ ). 